\title{
Recovery Failure Probability of Power-based NOMA on the Uplink of a 5G Cell for an Arbitrary Number of Superimposed Signals
}

\author{
Maria Luisa Merani \\ Dipartimento di Ingegneria "Enzo Ferrari" \\ University of Modena and Reggio Emilia, Modena, Italy \\ e-mail:marialuisa.merani@unimore.it
}

\begin{abstract}
This work puts forth an analytical approach to evaluate the recovery failure probability of powerbased Non-Orthogonal Multiple Access (NOMA) on the uplink of a 5G cell, the recovery failure being defined as the unfortunate event where the receiver is unable to decode even one out of the $n$ simultaneously received signals. In the examined scenario, Successive Interference Cancellation (SIC) is considered and an arbitrary number of superimposed signals is present. For the Rayleigh fading case, the recovery failure probability is provided in closed-form, clearly outlining its dependency on the signal-to-noise ratio of the users that are simultaneously transmitting, as well as on their distance from the receiver.
\end{abstract}

keywords - Uplink NOMA, successive interference cancellation, recovery failure probability, 5G

\section{INTRODUCTION}

In recent years NOMA has stirred much interest, because of its promise of enhancing the capacity of $5 \mathrm{G}$ cellular systems. NOMA allows different simultaneous users to share the available system resources (frequency, time) through a variety of different techniques, well illustrated in [1] and [2]: as a matter of fact, NOMA can operate in the power-domain, can adopt spreading sequences, can rely on coding matrices and/or interleaving. This work focuses on power-based NOMA on the uplink of a $5 \mathrm{G}$ cell, when a SIC receiver is employed. In [3], emphasis was on uplink power-based NOMA too, the goal being to evaluate the achievable sum data rate and the corresponding outage, which was provided in closed-form for the case of two users. The latter condition is commonly encountered in literature, as it guarantees a tractable analysis. Unlike [3] and previous works, this study sets no limit to the number of superimposed signals. Its main original contribution consists in the introduction of an analytical framework to evaluate the recovery failure probability, which is defined as the probability that the recovery of the signals simultaneously arriving at the receiver fails. In other words, not even one out of the $n$ received signals can be successfully decoded; this condition happens when the constraints on the received powers that the SIC receiver mandates are not observed. For the case of Rayleigh fading, the theoretical analysis provides the recovery failure probability in closed-form, immediately revealing its dependency on several factors, among which frequency, different signal-to-noise ratio assignments and distance of the users from the receiver. The numerical results explore the setting where either two or three superimposed signals are present, and show that several configurations exist, where the recovery failure probability is confined to low values and the benefit of NOMA can indeed be effectively exploited.

The remainder of this manuscript is organized as follows: Section II defines the scenario of investigation and develops the theoretical analysis; the case of Rayleigh fading is illustrated in Section III; Section IV provides some reference results, while the conclusions are drawn in Section V.

\section{Scenario And Performance Analysis}

Within the current work, uplink communications in a $5 \mathrm{G}$ cell are examined; power-based NOMA is employed and the reference scenario features $n$ User 
Equipments ( $U E \mathrm{~s}$ ) that transmit to the enhanced node B (enodeB), on the same radio spectrum. Let $h_{i}$ denote the envelope of the channel between the $i$-th $U E, U E_{i}, i=1,2, \ldots, n$, and the enodeB; as in [4], let

$$
\Gamma_{i}=\frac{h_{i}^{2}}{N_{0} B}
$$

indicate the $i$-th normalized channel gain, $N_{0}$ being the noise power spectral density and $B$ the transmission bandwidth. Further, let $p_{\Gamma_{i}}\left(\gamma_{i}\right)$ be the probability density function (pdf) of the generic $\Gamma_{i}$, $0 \leq \gamma_{i} \leq+\infty$. The assumption is that, $\forall i$ and $j$, $\Gamma_{i}$ and $\Gamma_{j}$ be independent random variables with different mean values, that is to say, different $U E \mathrm{~s}$ experience independent channel conditions during their transmissions to the enodeB.

Unlike LTE, 5G uplink power-based NOMA mandates for different transmit power levels for different $U E \mathrm{~s}$, in order to guarantee distinct received powers at the enodeB and to facilitate the task of the interference cancellation receiver. Moreover, unlike downlink power-based NOMA [5], which assigns users with stronger channel conditions lower power levels, for uplink NOMA the more favorable the channel gain that the $U E$ experiences, the higher the transmit power level that the $U E$ has to work with. In other words, before NOMA $U E$ s transmit, the enodeB ranks them according to their channel gains, strongest first; then, the $U E$ with the largest channel gain is assigned the highest transmit power, the $U E$ with the second largest gain the second highest power, until the least favored $U E$, which operates with the lowest transmit power level.

No matter what power assignment law is adopted, the instantaneous values of the $\Gamma_{i} \mathrm{~s}$ need to be ordered, so as to obtain $\Gamma_{(1)}, \Gamma_{(2)}, \ldots, \Gamma_{(n)}$, with

$$
\Gamma_{(1)}>\Gamma_{(2)}>\ldots>\Gamma_{(n)} ;
$$

next, transmit powers are assigned to $U E$ s respecting the constraint

$$
P_{(1)}>P_{(2)}>\ldots>P_{(n)},
$$

$P_{(i)}$ representing the transmit power of $U E_{(i)}$, the $U E$ with the $i$-th largest channel gain $\Gamma_{(i)}$.

Note that in the ordered set $\Gamma_{(1)}, \Gamma_{(2)}, \ldots, \Gamma_{(n)}$ the new random variables $\Gamma_{(i)}$ s are no longer independent and that, for the notation employed, $\Gamma_{(1)}$ is the largest order statistic, whereas $\Gamma_{(n)}$ is the smallest.
The enodeB receives the superimposed messages from the $U E$ s and through successive interference cancellation it attempts to decode them: if totally successful, it recovers all $n$ signals, the strongest first, then the second strongest, until the weakest.

We begin observing that the strongest signal received by the enodeB from $U E_{(1)}$ can be detected if the difference between the power received from $U E_{(1)}$ and the sum of the powers received from all other $U E \mathrm{~s}$ is greater than or equal to a threshold value $P_{\text {thres }}$, that represents the minimum difference required to extract the first signal. In analytical terms, the condition of successful recovery for such signal is given by

$$
P_{(1)} \Gamma_{(1)}-\sum_{i=2}^{n} P_{(i)} \Gamma_{(i)} \geq P_{\text {thres }} .
$$

If (4) is satisfied, then the first decoding event occurs, the SIC receiver recovers one signal and next proceeds and attempts to decode the second strongest signal, received from $U E_{(2)}$. The first signal is subtracted out, so that the recovery of the second strongest received signal requires the condition

$$
P_{(2)} \Gamma_{(2)}-\sum_{i=3}^{n} P_{(i)} \Gamma_{(i)} \geq P_{\text {thres }}
$$

to hold. If (5) is verified, then the receiver decodes two signals and can tackle the decoding of the third strongest signal; on the contrary, if last condition is not fulfilled, the SIC receiver halts. In general, the process stops when either the last, weakest signal is successfully decoded, or when $k$ signals only, $k<$ $n-1$, are recovered, this event corresponding to the occurrence of the condition:

$$
P_{(k+1)} \Gamma_{(k+1)}-\sum_{i=k+2}^{n} P_{(i)} \Gamma_{(i)}<P_{\text {thres }} .
$$

The final consequence of this iterated reasoning is that inequality (4) represents the necessary and sufficient condition for the decoding of the strongest signal, but also the necessary condition for the decoding of $2,3, \ldots, n$ signals out of $n$.

Next, let us define $P_{f_{a i l}}$, the recovery failure probability of power-based uplink NOMA in the presence of $n$ simultaneous transmissions, as the probability that not even one, out of the $n$ superimposed signals, can be correctly recovered by the 
SIC receiver. From the previous reasoning it follows that

$$
P_{\text {fail }_{n}}=
$$

$=1-\operatorname{Pr}\{$ the strongest signal is recovered $\}=$

$$
=\operatorname{Pr}\left\{P_{(1)} \Gamma_{(1)}-\sum_{i=2}^{n} P_{(i)} \Gamma_{(i)}<P_{\text {thres }}\right\} \text {. }
$$

Evaluating (7) requires the consideration of $n$ dependent random variables, the generic of which is

$$
X_{(i)}=P_{(i)} \Gamma_{(i)} \text {; }
$$

recalling (2) and (3), it is immediate to conclude that condition $X_{(1)}>X_{(2)}>\ldots>X_{(n)}$ holds.

To understand how the $P_{f_{a i l}}$ evaluation can be pursued, it is instructive to consider $n=2$ and to indicate by $f_{\text {joint }_{2}}\left(x_{(1)}, x_{(2)}\right)$ the joint pdf of the random variables $X_{(1)}$ and $X_{(2)}$, so that the recovery failure probability in (7) becomes

$$
\begin{gathered}
P_{\text {fail }_{2}}=\operatorname{Pr}\left\{P_{(1)} \Gamma_{(1)}-P_{(2)} \Gamma_{(2)}<P_{\text {thres }}\right\}= \\
=\operatorname{Pr}\left\{X_{(1)}-X_{(2)}<P_{\text {thres }}\right\}= \\
=1-\operatorname{Pr}\left\{X_{(2)}<X_{(1)}-P_{\text {thres }}\right\}= \\
=1-\int_{x_{(1)}=P_{\text {thres }}}^{\infty} \int_{x_{(2)}=0}^{x_{(1)}-P_{\text {thres }}} f_{\text {joint }_{2}}\left(x_{(1)}, x_{(2)}\right) . \\
\cdot d x_{(2)} d x_{(1)} .
\end{gathered}
$$

For a generic $n$, last expression generalizes to

$$
\begin{gathered}
P_{\text {fail }_{n}}=1- \\
\int_{x_{(1)}=P_{\text {thres }}}^{\infty} \int_{x_{(2)}=0}^{x_{(1)}-P_{\text {thres }}} \int_{x_{(3)}=0}^{x_{(1)}-x_{(2)}-P_{\text {thres }}} \ldots \\
\int_{x_{(n)}=0}^{x_{(1)}-x_{(2)}-x_{(3)}-\ldots-x_{(n-1)}-P_{\text {thres }}} \\
f_{\text {joint }_{n}}\left(x_{(1)}, x_{(2)}, \ldots, x_{(n)}\right) \cdot \\
\cdot d x_{(n)} d x_{(n-1)} \ldots d x_{(1)},
\end{gathered}
$$

where $f_{\text {joint }_{n}}\left(x_{(1)}, x_{(2)}, \ldots, x_{(n)}\right)$ indicates the joint pdf of the ordered set $X_{(1)}, X_{(2)}, \ldots, X_{(n)}$.

The next problem at hand is to determine such pdf. In this respect, let $f_{i}(\cdot)$ be the pdf of the unordered - random variable $X_{i}$, defined as

$$
X_{i}=P_{(i)} \cdot \Gamma_{i}, \quad i=1,2, \ldots, n,
$$

whose pdf is immediately determined, once $p_{\Gamma_{i}}\left(\gamma_{i}\right)$ is known, as $P_{(i)}$ is a constant, and define $F_{n}$ as the following $n \times n$ matrix

$$
F_{n}=\left[\begin{array}{llll}
f_{1}\left(x_{(1)}\right) & f_{2}\left(x_{(1)}\right) & \ldots & f_{n}\left(x_{(1)}\right) \\
f_{1}\left(x_{(2)}\right) & f_{2}\left(x_{(2)}\right) & \ldots & f_{n}\left(x_{(2)}\right) \\
\vdots & \vdots & \ddots & \vdots \\
f_{1}\left(x_{(n)}\right) & f_{2}\left(x_{(n)}\right) & \ldots & f_{n}\left(x_{(n)}\right)
\end{array}\right]
$$

For the purpose of what follows, recall that the permanent of a square matrix $A$, written as $\left.\right|^{+} A+$, is defined like the determinant, except that all signs are positive. Again, to make things clearer, consider the case $n=2$ and observe that in this case the permanent of $F_{2}$ is given by:

$$
\begin{aligned}
& +F_{2} \stackrel{+}{\mid}=+\left[\begin{array}{ll}
f_{1}\left(x_{(1)}\right) & f_{2}\left(x_{(1)}\right) \\
f_{1}\left(x_{(2)}\right) & f_{2}\left(x_{(2)}\right)
\end{array}\right]+ \\
& =f_{1}\left(x_{(1)} f_{2}\left(x_{(2)}\right)+f_{2}\left(x_{(1)}\right) f_{1}\left(x_{(2)}\right) .\right.
\end{aligned}
$$

For an arbitrary $n$, it can be demonstrated that the joint pdf $f_{\text {joint }_{n}}\left(x_{(1)}, x_{(2)}, \ldots, x_{(n)}\right)$ of the ordered statistics $X_{(1)}, X_{(2)}, \ldots, X_{(n)}$ is

$$
f_{\text {joint }_{n}}\left(x_{(1)}, x_{(2)}, \ldots, x_{(n)}\right)=\stackrel{+}{\mid} F_{n} \stackrel{+}{\mid},
$$

$F_{n}$ being given by (12). Last result is substantiated by the reasoning in [6] and [7], where the arguments of [8] are extended to prove the formulation in (14) with the use of permanents.

At first sight, $f_{\text {joint }_{n}}\left(x_{(1)}, x_{(2)}, \ldots, x_{(n)}\right)$ gives the impression that evaluating the integral in (10) might be quite cumbersome when $n$ is arbitrary. However, the joint pdf obeys a highly peculiar structure and an alike - and more convenient - rewriting of it is provided in the following terms: let $\mathcal{S}_{\mathcal{N}}$ indicate all $n$ ! permutations of the set $N=\{1,2, \ldots, n\}$ and by $S_{i}=\left\{i_{1}, i_{2}, \ldots, i_{n}\right\}$ the generic of such permutations. It follows that $f_{\text {joint }_{n}}\left(x_{(1)}, x_{(2)}, \ldots, x_{(n)}\right)$ is equivalently written as

$$
\begin{array}{r}
f_{\text {joint }_{n}}\left(x_{(1)}, x_{(2)}, \ldots, x_{(n)}\right)= \\
=\sum_{S_{i} \in \mathcal{S}_{\mathcal{N}}} f_{1}\left(x_{\left(i_{1}\right)}\right) f_{2}\left(x_{\left(i_{2}\right)}\right) \cdot \ldots \cdot f_{n}\left(x_{\left(i_{n}\right)}\right) .
\end{array}
$$

Last expression highlights that the joint pdf exhibits the presence of $n$ ! terms, wherein the permutations of the arguments of the $f_{1}(\cdot), f_{2}(\cdot), \ldots, f_{n}(\cdot)$ pdfs appear. Replacing (15) in (10) gives $P_{f_{a i l} n}$ as

$$
\begin{aligned}
& P_{\text {fail }_{n}}=1- \\
& \int_{x_{(1)}=P_{\text {thres }}}^{\infty} \int_{x_{(2)}=0}^{x_{(1)}-P_{\text {thres }}} \int_{x_{(3)}=0}^{x_{(1)}-x_{(2)}-P_{\text {thres }}} \ldots \\
& \int_{x_{(n)}=0}^{x_{(1)}-x_{(2)}-x_{(3)}-\ldots-x_{(n-1)}-P_{\text {thres }}} \\
& \sum_{S_{i} \in \mathcal{S}_{\mathcal{N}}} f_{1}\left(x_{\left(i_{1}\right)}\right) f_{2}\left(x_{\left(i_{2}\right)}\right) \cdot \ldots \cdot f_{n}\left(x_{\left(i_{n}\right)}\right) \cdot \\
& \cdot d x_{(n)} d x_{(n-1)} \ldots d x_{(1)},
\end{aligned}
$$


and indicating by $I_{S_{i}}$ the result of the integral

$$
\begin{aligned}
& I_{S_{i}}=\int_{x_{(1)}=P_{\text {thres }}}^{\infty} \int_{x_{(2)}=0}^{x_{(1)}-P_{\text {thres }}} \int_{x_{(3)}=0}^{x_{(1)}-x_{(2)}-P_{\text {thres }}} \ldots \\
& \begin{array}{l}
x_{(1)}-x_{(2)}-x_{(3)}-\ldots-x_{(n-1)}-P_{\text {thres }} \\
\int_{(n)}=0
\end{array} \\
& p_{i_{1} i_{2} \ldots i_{n}}\left(x_{(1)}, x_{(2)}, \ldots, x_{(n)}\right) . \\
& \cdot d x_{(n)} d x_{(n-1)} \ldots d x_{(1)} \text {, }
\end{aligned}
$$

where

$$
\begin{array}{r}
p_{i_{1} i_{2} \ldots i_{n}}\left(x_{(1)}, x_{(2)}, \ldots, x_{(n)}\right)= \\
f_{1}\left(x_{\left(i_{1}\right)}\right) f_{2}\left(x_{\left(i_{2}\right)}\right) \cdot \ldots \cdot f_{n}\left(x_{\left(i_{n}\right)}\right),
\end{array}
$$

then $P_{\text {fail }_{n}}$ is rewritten as

$$
P_{\text {fail }_{n}}=1-\sum_{S_{i} \in \mathcal{S}_{\mathcal{N}}} I_{S_{i}} .
$$

Luckily, when the random variables $X_{1}, X_{2}, \ldots$, $X_{n}$ obey the same statistical description, although with different mean values, for a permutation $S_{j}$ different than $S_{i}$, the $I_{S_{j}}$ result is readily obtained from $I_{S_{i}}$ through the analogous permutation of the $f_{i}(\cdot)$ 's arguments $x_{\left(i_{k}\right)}, k=1,2, \ldots, n$, in (18), $\forall j$. That is to say, given the $n$-th fold integral in (17) has been solved once, e.g., $I_{S_{1}}$ has been determined, $S_{1}=\{1,2, \ldots, n\}$, then all the remaining $I_{S_{i}}$ terms are known. This significantly reduces $P_{\text {fail }_{n}}$ computational complexity in $n$, no matter what statistical description is examined for $\Gamma_{i}, i=1,2, \ldots, n$.

As an illustrative example, the case of Rayleigh fading is examined in next Section.

\section{RAYLEIGH FADING CASE}

When the envelope of the received signal is subject to Rayleigh fading, $\Gamma_{i}$ and in turn $X_{i}$ are exponentially distributed with means $\bar{\Gamma}_{i}$ and $\bar{X}_{i}$, respectively. Beginning with the case $n=2$, from (19) $P_{\text {fail }_{2}}$ specializes to

$$
P_{\text {fail }_{2}}=1-\left(I_{S_{1}}+I_{S_{2}}\right),
$$

where $S_{1}=\{1,2\}$ and

$$
I_{S_{1}}=\int_{x_{(1)}=P_{\text {thres }}}^{\infty} \int_{x_{(2)}=0}^{x_{(1)}-P_{\text {thres }}} p_{12}\left(x_{(1)}, x_{(2)}\right) d x_{(2)} d x_{(1)}
$$

with

$$
\begin{array}{r}
p_{12}\left(x_{(1)}, x_{(2)}\right)=f_{1}\left(x_{(1)}\right) f_{2}\left(x_{(2)}\right)= \\
=\frac{1}{\bar{X}_{1}} \exp \left(-\frac{x_{(1)}}{\bar{X}_{1}}\right) \cdot \frac{1}{\bar{X}_{2}} \exp \left(-\frac{x_{(2)}}{\bar{X}_{2}}\right) ;
\end{array}
$$

analogously, $S_{2}=\{2,1\}$ and

$$
I_{S_{2}}=\int_{x_{(1)}=P_{\text {thres }}}^{\infty} \int_{x_{(2)}=0}^{x_{1}-P_{\text {thres }}} p_{21}\left(x_{(1)}, x_{(2)}\right) d x_{(2)} d x_{(1)}
$$

with

$$
\begin{gathered}
p_{21}\left(x_{(1)}, x_{(2)}\right)=f_{1}\left(x_{(2)}\right) f_{2}\left(x_{(1)}\right)= \\
=\frac{1}{\bar{X}_{1}} \exp \left(-\frac{x_{(2)}}{\bar{X}_{1}}\right) \cdot \frac{1}{\bar{X}_{2}} \exp \left(-\frac{x_{(1)}}{\bar{X}_{2}}\right) .
\end{gathered}
$$

Solving the integral in (21) gives

$$
I_{S_{1}}=\frac{\bar{X}_{1}}{\bar{X}_{1}+\bar{X}_{2}} \exp \left(-\frac{P_{\text {thres }}}{\bar{X}_{1}}\right),
$$

wherefore $I_{S_{2}}$ immediately follows as

$$
I_{S_{2}}=\frac{\bar{X}_{2}}{\bar{X}_{2}+\bar{X}_{1}} \exp \left(-\frac{P_{\text {thres }}}{\bar{X}_{2}}\right),
$$

and finally

$$
\begin{gathered}
P_{\text {fail }_{2}}=1+ \\
-\left(\frac{\bar{X}_{1}}{\bar{X}_{1}+\bar{X}_{2}} \exp \left(\frac{-P_{\text {thres }}}{\bar{X}_{1}}\right)+\frac{\bar{X}_{2}}{\bar{X}_{1}+\bar{X}_{2}} \exp \left(\frac{-P_{\text {thres }}}{\bar{X}_{2}}\right)\right) .
\end{gathered}
$$

Similarly, when $n=3$, there will be 3 ! distinct integral contributions of the type in (17) in the recovery failure probability expression, that are determined once $p_{123}\left(x_{(1)}, x_{(2)}, x_{(3)}\right)$ is introduced,

$$
\begin{gathered}
p_{123}\left(x_{(1)}, x_{(2)}, x_{(3)}\right)= \\
\frac{1}{\overline{X_{1}}} \exp \left(-\frac{x_{(1)}}{\bar{X}_{1}}\right) \cdot \frac{1}{\bar{X}_{2}} \exp \left(-\frac{x_{(2)}}{\bar{X}_{2}}\right) . \\
\cdot \frac{1}{\bar{X}_{3}} \exp \left(-\frac{x_{(3)}}{\bar{X}_{3}}\right) .
\end{gathered}
$$

Now, $S_{1}=\{1,2,3\}$ and

$$
\begin{gathered}
I_{S_{1}}=\int_{x_{(1)}=}^{\infty} P_{\text {thres }} \int_{x_{(2)}=0}^{x_{(1)}-P_{\text {thres }}} \int_{x_{(3)}=0}^{x_{(1)}-x_{(2)}-P_{\text {thres }}} \\
p_{123}\left(x_{(1)}, x_{(2)}, x_{(3)}\right) \cdot \\
\cdot d x_{(3)} d x_{(2)} d x_{(1)},
\end{gathered}
$$

that after a few passages is determined as

$$
I_{S_{1}}=\frac{{\overline{X_{1}}}^{2} \exp \left(-\frac{P_{\text {thres }}}{\overline{X_{1}}}\right)}{\left(\overline{X_{1}}+\overline{X_{2}}\right)\left(\overline{X_{1}}+\overline{X_{3}}\right)} .
$$

Moreover, it can be verified that the set $S_{2}=$ $\{1,3,2\}$ gives the same result as $S_{1}$, that is to say, $I_{S_{1}}=I_{S_{2}}$. If we now introduce $S_{3}=\{2,1,3\}$ and $S_{4}=\{2,3,1\}$, suitably permuting $\bar{X}_{1}, \bar{X}_{2}$ and $\bar{X}_{3}$ in (30) $I_{S_{3}}=I_{S_{4}}$ is also determined; the same applies 
to $S_{5}=\{3,2,1\}$ and $S_{6}=\{3,1,2\}$, for which $I_{S_{5}}=I_{S_{6}} \cdot P_{f_{a i l}}$ is then provided as

$$
\begin{array}{r}
P_{\text {fail }_{3}}=1-\sum_{S_{i} \in S_{N}} I_{S_{i}}= \\
1-2\left(\frac{\bar{X}_{1}^{2} \exp \left(-\frac{P_{\text {thres }}}{\bar{X}_{1}}\right)}{\left(\bar{X}_{1}+\bar{X}_{2}\right)\left(\bar{X}_{1}+\bar{X}_{3}\right)}+\right. \\
+\frac{\bar{X}_{2}^{2} \exp \left(-\frac{P_{\text {thres }}}{\bar{X}_{2}}\right)}{\left(\bar{X}_{2}+\bar{X}_{1}\right)\left(\bar{X}_{2}+\bar{X}_{3}\right)}+ \\
\left.+\frac{\bar{X}_{3}^{2} \exp \left(-\frac{P_{\text {thres }}}{\bar{X}_{3}}\right)}{\left(\bar{X}_{3}+\bar{X}_{2}\right)\left(\bar{X}_{3}+\bar{X}_{1}\right)}\right) .
\end{array}
$$

Iterating the procedure, by induction it is proved that $P_{f_{a i l}}$, the recovery failure probability in the presence of $n$ superimposed signals, is given in closed form by:

$$
\begin{aligned}
P_{\text {fail }_{n}} & =1-\frac{n !}{n} \cdot \sum_{k=1}^{n} \frac{\bar{X}_{k}^{n-1} e^{-\frac{P_{\text {thresh }}}{\bar{X}_{k}}}}{\prod_{\substack{i=1 \\
i \neq k}}^{n}\left(\bar{X}_{k}+\bar{X}_{i}\right)}= \\
& =1-(n-1) ! \sum_{k=1}^{n} \frac{e^{-\frac{P_{\text {thres }}}{\bar{X}_{k}}}}{\prod_{\substack{i=1 \\
i \neq k}}^{n}\left(1+\frac{\bar{X}_{i}}{\bar{X}_{k}}\right)} .
\end{aligned}
$$

Last expression allows to determine the recovery failure probability in the presence of an arbitrary number of signals in a very effective and quick manner. To this regard, from (11) and (1) it is observed that

$$
\bar{X}_{i}=P_{(i)} \bar{\Gamma}_{i}=\frac{P_{(i)}}{N_{0} B} \cdot \overline{h_{i}^{2}}=S N R_{(i)} \cdot \overline{h_{i}^{2}}
$$

where $S N R_{(i)}$ is the signal-to-noise ratio of $U E_{(i)}$ and $\overline{h_{i}^{2}}$ is the mean of the channel gain $h_{i}^{2}$. Hence, when the $S N R_{(i)}$ and the $\overline{h_{i}^{2}}$ values are provided, the recovery failure probability of uplink power-based NOMA is known. We next assume that the path loss is

$$
\overline{h_{i}^{2}}=k_{p} \cdot D_{i}^{-\alpha},
$$

where $D_{i}$ is the distance between the $i$-th $U E$ and the enodeB, $\alpha$ represents the decay factor and $k_{p}$ is $k_{p}=\left(\frac{c}{4 \pi f_{c}}\right)^{2}, c$ being the speed of light and $f_{c}$ the operating frequency; moreover, isotropic antennas are considered. In this circumstance, (32) specializes to

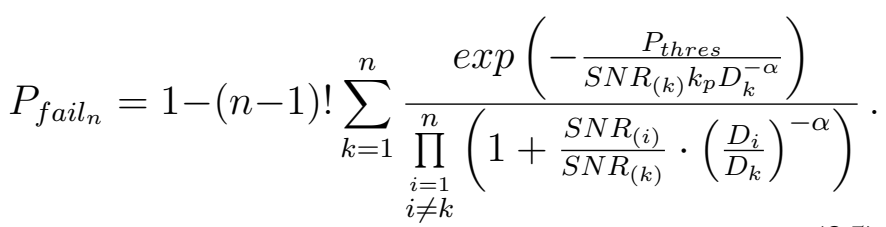

Given $P_{\text {thres }}$ is fixed, as well as the operating frequency $f_{c}$, the set of distances $D_{1}, D_{2}, \ldots$, $D_{n}$ and the SNR values $S N R_{(1)}, S N R_{(2)}, \ldots$, $S N R_{(n)}$, from (35) the probability of not being able to take advantage of successive interference cancellation is determined right away. Next Section relies on (35) to offer some meaningful insights on the performance of uplink power-based NOMA employed in conjunction with SIC.

\section{NumericAl Results}

Fig.1 reports $P_{f_{a i l}}$, the recovery failure probability in the presence of two superimposed signals, as a function of $D_{1}$, the distance of $U E_{1}$ from the enodeB given in meters, when the second $U E, U E_{2}$, is at the cell edge and the cell radius is $R=100 \mathrm{~m}$; $S N R_{(1)}$ takes on different values, namely, $=15,12$ and $10 \mathrm{~dB}$, whereas $S N R_{(2)}=10 \mathrm{~dB}$, that is to say, a difference of 5,2 and $0 \mathrm{~dB}$ between the transmitted powers of the two $U E$ s is considered. This is in line with the choices performed in [3], where the transmitted powers of two simultaneous users differ for either 5 or $3 \mathrm{~dB}$. The propagation factor is $\alpha=4$ and two values of the carrier frequency are considered: $f_{c}=2 \mathrm{GHz}$ (solid lines) and $28 \mathrm{GHz}$ (dashed lines). $P_{\text {thres }}$, the minimum difference in received powers is equal to $-75 \mathrm{dBm}$ [9]. The frequency effect on the recovery failure probability is evident, highlighting that power-based NOMA is by far more attractive at lower frequencies. Nevertheless, interesting recovery failure probability values can be attained when the distance of $U E_{1}$ from the enodeB is small and the gap between $S N R_{(1)}$ and $S N R_{(2)}$ increases. Fig.2 extends the reasoning to the case of three superimposed signals and shows the behavior of the recovery failure probability $P_{f_{a i l}}$ as a function of $D_{1}$ for three distinct choices of the $\left(S N R_{(1)}, S N R_{(2)}, S N R_{(3)}\right)$ triplet, namely: $(10,10,10)$ (solid lines), $(12,10,10)$ (dashed lines) and $(15,10,8)$ (dotted lines), when the carrier frequency is $f_{c}=28 \mathrm{GHz}$. Different locations of $U E_{2}$ 


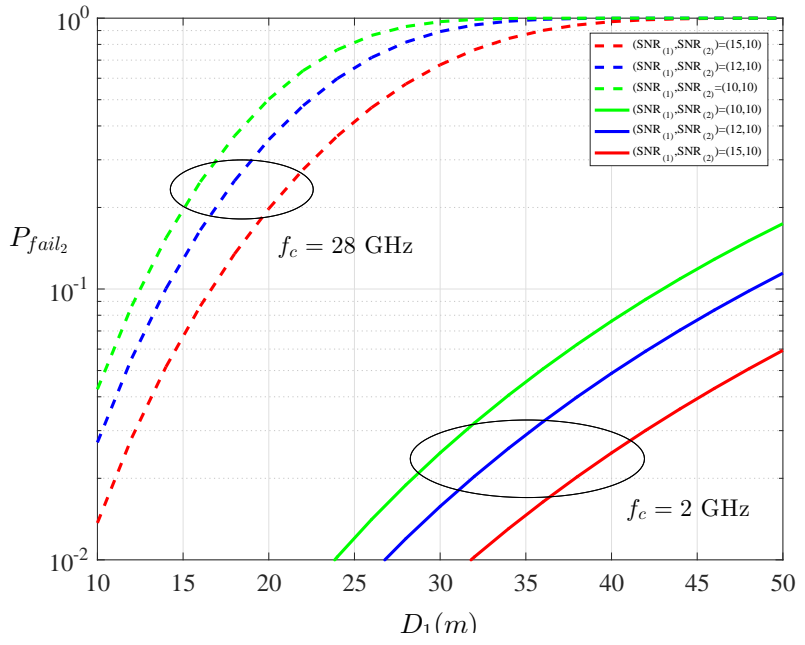

Fig. 1. $P_{f_{a i l_{2}}}$ as a function of $D_{1}, f_{c}=2$ and $f_{c}=28 \mathrm{GHz}$

and $U E_{3}$ are examined: $D_{2}=0.2 R$ paired with $D_{3}=0.5 R$ (red lines), $D_{2}=0.2 R$ with $D_{3}=0.7 R$ (blue lines), and $D_{2}=0.2 R$ with $D_{3}=0.9 R$ (green lines). The curves indicate that the influence of $D_{3}$ on $P_{f_{a i l} 3}$ is modest: in the examined setting, it is $U E_{2}$ that plays the lion's share, being close to the enodeB, as $D_{2}=0.2 R$ reveals. All curves exhibit a similar shape; however, they become wider for more pronounced differences in the $S N R \mathrm{~s}$, whose final effect is to increase the range of $D_{1}$ values for which the recovery failure probability stays below a predefined threshold (e.g., $10^{-1}$ ). So, the advantage of markedly separating the $U E \mathrm{~s}$ in terms of $S N R$, assigning the users with the most favorable channel a higher $S N R$ value, is manifest and numerically quantified. Moreover, note that in the majority of the cases $P_{\mathrm{fail}_{3}}$ values fall below $2 \cdot 10^{-1}$.

\section{CONCLUSIONS}

This paper has identified a novel, analytical method to determine the probability of not being able to take advantage of power-based NOMA on the uplink of a 5G cell, when successive interference cancellation is employed and an arbitrary number of superimposed signals is considered. As a representative example, Rayleigh fading has been examined and the corresponding recovery failure probability provided in closed-form. The dependency of the recovery failure probability on carrier frequency, signal-to-noise ratio of the $U E$ s that are simultaneously transmitting, as well as on their distance from

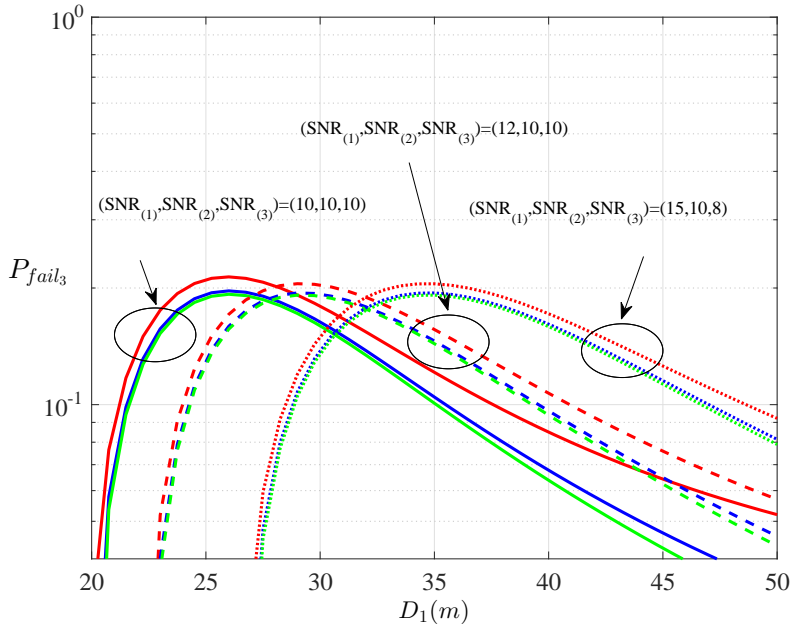

Fig. 2. $P_{\mathrm{fail}_{3}}$ as a function of $D_{1}, f_{c}=28 \mathrm{GHz}$

the enodeB has been clearly identified, revealing that even at very high frequencies there exist several operating regions where power-based NOMA combined with SIC exhibits notably low recovery failure probability values, in the presence of two and also three simultaneous users.

\section{REFERENCES}

[1] Y. Chunlin, Y. Zhifeng, L. Weimin, Y. Yifei, "Non-Orthogonal Multiple Access Schemes for 5G," ZTE Communications, Vol.14, no.4, pp.11-16, October 2016.

[2] L. Dai, B. Wang, Y. Yuan, S. Han, Chin-Li I, Z. Wang, "NonOrthogonal Multiple Access for 5G: Solutions, Challenges, Opportunities, and Future Research Trends," IEEE Communications Magazine, Vol.53, no.9, pp.84-81, September 2015.

[3] N. Zhang, J. Wang, G. Kang, Y. Liu, “Uplink Nonorthogonal Multiple Access in 5G Systems," IEEE Communications Letters, Vol. 20, no.3, pp.458-461, March 2016.

[4] S. Ali, H. Tabassum, E. Hossain, "Dynamic User Clustering and Power Allocation for Uplink and Downlink Non-Orthogonal Multiple Access (NOMA) Systems," IEEE Access, Special Section on Optimization for Emerging Wireless Networks: IoT, 5G and Smart Grid Communications, Vol.4, pp.6325-6343, October 2016.

[5] Z. Ding, M. Peng, H.V. Poor, "Cooperative Non-Orthogonal Multiple Access in 5G Systems," IEEE Comm. Letters, Vol.19, no.8, pp.1462-1465, August 2015.

[6] R.J. Vaughan, W.N. Venables, "Permanent Expressions for Order Statistics Densities," Journal of the Royal Statistical Society, Vol.34, no.2, pp. 308-31, 1972.

[7] R. Maffei, U. Manzoli, M.L. Merani, "RAKE reception with unequal power path signals", IEEE Trans. on Communications, Vol.52, no.1, pp.24-27, January 2004.

[8] M.G. Kendall, A. Stuart, "The Advanced Theory of Statistics", 2nd edition, Vol.1, 1958.

[9] T. S. Rappaport, S. Sun, R. Mayzus, H. Zhao, Y. Azar, K. Wang, G.N. Wong, J. K. Schulz, M. Samimi, F. Gutierrez, "Millimeter Wave Mobile Communications for 5G Cellular: It Will Work!," IEEE Access, Vol.1, pp.335-349, 2013. 